"downstream" RALDH enzymes. These data imply that RA availability is regulated differently in mice and man, with expression of $\mathrm{RDH} 10$ providing an important control point in humans.

Competing interests None declared.

\section{PWE-258 THIOPURINE MONITORING IN INFLAMMATORY BOWEL DISEASE PATIENTS AT A DISTRICT GENERAL HOSPITAL}

doi:10.1136/gutjnl-2012-302514d.258

${ }^{1} \mathrm{~T}$ Jowitt, ${ }^{*} \mathrm{C}$ Bowman, ${ }^{2} \mathrm{M}$ Ahmed, ${ }^{2} \mathrm{~S}$ Singh, ${ }^{2} \mathrm{C} \mathrm{H}$ Lim. ${ }^{1}$ Medical School, University of Birmingham, Birmingham, UK; ${ }^{2}$ Department of Gastroenterology, Good Hope Hospital, Sutton Coldfield, UK

Introduction Thiopurines are an unlicensed but recognised therapy for Inflammatory Bowel Disease (IBD). These drugs interfere with cell signalling and have significant side effects including leucopaenia, pancreatitis and hepatotoxicity. Therefore routine monitoring of blood is mandatory. Our Trust has guidelines (based on BSG guidelines) for the monitoring of Thiopurine therapy. Patients are monitored by a IBD Nurse specialist using a simple electronic database.

Methods Objective: To determine the effectiveness of the IBD Nurse database in ensuring that patients on Thiopurines are monitored according to Trust guidelines. A total of 900 patients with IBD attend our gastroenterology clinics. Of these 204 are on Thiopurines. Trust guidelines recommends weekly blood test monitoring for the first month starting Thiopurines. This is followed by monthly for the next 3 months and every 3 months subsequently. Patients latest blood results were collected over a two consecutive days in July 2011 for 204 patients. Patients more than 7 days late for blood tests were considered non-compliance with the guideline.

Results 182 (89\%) patients were having their bloods monitored as per trust guidelines. However 22 patients (11\%) were being monitored incorrectly, with a median of 68.5 (32.25-269.25) days overdue. 16 of these patients have a diagnosis of Crohn's Disease, the remaining six have Ulcerative Colitis. 14 (7\%) patients had either deranged liver function tests or were neutropaenic.

Conclusion The current manual database is reliable as the majority of thiopurines patients were being monitored as per hospital guideline. However, $7 \%$ of abnormal blood results may not be recognised promptly and no action was taken. An automated database with automated reminder to patients who passed their blood test due date and electronic notice to the doctor responsible for the patient is needed to reduce the potential risk of harm to the patients.

Competing interests None declared.

\section{REFERENCE}

1. Anon. Guidelines for the management of inflammatory bowel disease in adults. Gut 2011;60:571-607.

\section{PWE-259 THE EFFICACY OF METHOTREXATE IN CROHN'S DISEASE: A CLINICAL PERSPECTIVE}

doi:10.1136/gutjnl-2012-302514d.259

T T Gordon-Walker,* M W Stahl, M Groome, J Todd, N Reynolds, C Mowat. Department of Gastroenterology, Ninewells Hospital, Dundee, UK

Introduction Methotrexate (MTX) has been shown in clinical trials to be effective in the induction and maintenance of remission in Crohn's Disease (CD). It is predominantly used in patients intolerant of, or whose disease is refractory to, thiopurine treatment. It was our aim to examine, in a clinical setting, the efficacy and sideeffect profile of MTX in patients with CD.
Methods A retrospective audit was performed by casenote review of all patients with $\mathrm{CD}$ attending the clinical investigations unit at Ninewells Hospital to commence parenteral MTX during the period 1 January 2007 to 31 December 2010. A total of 53 patients were identified, for whom casenotes were available in 52 (26 male/26 female). Intramuscular MTX treatment was initiated at a dose of $25 \mathrm{mg}$ once weekly for 16 weeks, followed by oral MTX at a dose of $15 \mathrm{mg}$ once weekly. Clinical response by physician's global assessment was recorded at 3, 6 and 12 months. Clinical response was defined as complete response (absence of IBD symptoms or complete healing of fistulae); partial response (symptomatic improvement but ongoing symptoms); or no response (no improvement or deterioration from baseline). Relapse was defined as deterioration in symptoms necessitating additional medical or surgical intervention.

Results Median age of starting MTX was 32 (range 15-73). 51 of 52 patients had previously received thiopurine treatment. 12-month follow-up data were available for 48 patients. 34 patients were taking steroids at the time of initiating MTX. At 12 months, complete response was reported in 8 patients $(17 \%)$, partial response in 6 patients $(13 \%)$, no response in 21 patients $(44 \%)$ and drug withdrawal due to side effects in 13 patients (27\%). Relapse was reported in 17 patients (35\%). 10 patients (21\%) required additional medical therapy (steroids or biological therapy). Surgical intervention was required in seven patients (15\%). Steroid withdrawal at 1 -year, without recourse to biological or surgical therapy, was reported in only 11 of 34 patients $(32 \%)$. MTX-associated side effects were reported in 25 patients (48\%). Reported side effects included: LFT abnormalities (9), nausea (8), lymphopenia (5), lethargy (3) and mouth ulceration (1). Monitoring of FBC/LFTs was performed at $97 \%$ of scheduled weekly intervals for patients receiving parenteral MTX and at $74 \%$ of scheduled monthly intervals for patients on oral MTX.

Conclusion In this cohort, the clinical effectiveness of MTX in the induction and maintenance of remission of CD was limited. Only $29 \%$ of patients had either a complete or partial response to therapy at 1-year. The clinical effectiveness of MTX was limited by sideeffects in $27 \%$. On the basis of these results we should re-consider the position of MTX in the management of CD

Competing interests None declared.

\section{PWE-260 OPTIMAL C REACTIVE PROTEIN CUT-OFF POINT FOR PREDICTING HOSPITALISATION IN PATIENTS WITH MODERATELY ACTIVE CROHN'S DISEASE}

doi:10.1136/gutjnl-2012-302514d.260

${ }^{1} \mathrm{~J}$-F Colombel, ${ }^{2} \mathrm{~W}$ J Sandborn, ${ }^{3} \mathrm{E}$ Louis, ${ }^{4} \mathrm{R}$ Panaccione, ${ }^{5} \mathrm{R}$ B Thakkar, ${ }^{5} \mathrm{M}$ M Castillo, ${ }^{5} \mathrm{M}$ Yang, ${ }^{6} \mathrm{~T}$ Finney-Hayward, ${ }^{*} \mathrm{~J}_{\mathrm{J}} \mathrm{Chao},{ }^{5} \mathrm{P} \mathrm{M}$ Mulani. ${ }^{1}$ Centre Hospitalier Universitaire de Lille, Lille, France; ${ }^{2}$ UCSD, La Jolla, California, USA; ${ }^{3} U$ of Liège, Liège, Belgium; ${ }^{4} U$ of Calgary, Calgary, Alberta, Canada; ${ }^{5}$ Abbott Laboratories, Abbott Park, Illinois, USA; ${ }^{6}$ Abbott Laboratories Ltd, Maidenhead, Berkshire, UK

Introduction To identify high risk patients among patients with moderate Crohn's disease (CD), we explored the association between $C$ reactive protein (CRP) concentration and hospitalisation risk for patients with moderately active CD and identified the optimal CRP cut-off point as a marker to predict CD-related hospitalisation. CRP is a well-studied and commonly used laboratory marker of inflammation in CD. ${ }^{1}$ The relationship between CRP and hospitalisation risk given the same Crohn's Disease Activity Impairment (CDAI) score in patients with moderate CD has not been studied.

Methods Data from CHARM, a 56-week (wk), randomised, placebocontrolled trial of adalimumab maintenance therapy, were analysed. All patients received adalimumab during a 4-wk, open-label induction period; patients were then randomised to adalimumab or placebo for a 52 -wk double-blind period. For this analysis, only patients who were randomised to placebo at Wk 4 and had 